\title{
Heat flux management via advanced magnetic divertor configurations and divertor detachment
}

\author{
E. Kolemen ${ }^{\mathrm{a} *}$, S.L. Allen ${ }^{\mathrm{b}}$, B.D. Bray ${ }^{\mathrm{c}}$, M.E. Fenstermacher ${ }^{\mathrm{b}}$, D.A. Humphreys ${ }^{\mathrm{c}}$, \\ A.W. Hyatt ${ }^{\mathrm{c}}$, C.J. Lasnier ${ }^{\mathrm{b}}$, A.W. Leonard ${ }^{\mathrm{c}}$, M.A. Makowski ${ }^{\mathrm{b}}$, A.G. McLean ${ }^{\mathrm{b}}$, R. Maingi $^{\mathrm{e}}$, \\ R. Nazikian ${ }^{\mathrm{e}}$, T.W. Petrie ${ }^{\mathrm{c}}$, V.A. Soukhanovskii ${ }^{\mathrm{b}}$, and E.A. Unterberg ${ }^{\mathrm{d}}$ \\ ${ }^{a}$ Princeton University, Princeton, New Jersey, 08544, USA \\ ${ }^{b}$ Lawrence Livermore National Laboratory, Livermore, California 94550, USA \\ ${ }^{c}$ General Atomics, PO Box 85608, San Diego, California 92186-5608 USA \\ ${ }^{d}$ Oak Ridge National Laboratory, PO Box 2008, Oak Ridge, Tennessee 37831 USA \\ ${ }^{e}$ Princeton Plasma Physics Laboratory, Princeton, New Jersey 08543, USA
}

\begin{abstract}
The Snowflake Divertor (SFD) control and detachment control to manage the heat flux at the divertor are successfully demonstrated at DIII-D. Results of the development and implementation of these two heat flux reduction control methods are presented. The SFD control algorithm calculates the position of the two null-points in real-time and controls shaping coil currents to achieve and stabilize various snowflake configurations. Detachment control stabilizes the detachment front fixed at specified distance between the strike point and the X-point throughout the shot.
\end{abstract}

PACS: 2.55.Fa 52.55.Rk 28.52.Fa

PSI-20 Keywords: Control, Heat flux, Snowflake, Detachment, Partial Detachment, Advanced Magnetic Divertor

*Corresponding and presenting author address: E. Kolemen, D302D, Engineering Quadrangle, Olden St, Princeton, NJ 08544, USA

Corresponding and presenting author e-mail: ekolemen@ @rinceton.edu 


\section{Snowflake divertor control}

The present vision of the tokamak plasma-material interface is an axisymmetric magnetic $\mathrm{X}$-point divertor. One approach to handling the high heat exhaust per unit area on the plasma facing components (PFCs) is to use alternative magnetic configurations. Examples of these advanced divertors are the snowflake divertor (SFD) [1], X-divertor [2], super X-divertor [3], and X-point target divertor [4]. These configurations require active and precise control of the magnetic configuration in order to regulate the particle and heat flow.

Recent research at DIII-D focused on the SFD configuration, which uses a second-order poloidal field null created by merging, or bringing close to each other, two first-order poloidal field null points (X-points) of a standard divertor configuration. A poloidal crosssection of the obtained magnetic flux surfaces with a hexagonal null-point has the appearance of a snowflake. The SFD geometry results in high poloidal flux expansion and a large plasma-wetted area compared to the standard divertor, which reduces peak heat flux. Also, SFD has four strike points which help share the divertor power load, compared to the twostrike-point configuration.

The exact second-order null configuration is topologically unstable to variations in coil currents, which destroy the perfect alignment. This splits the double null into two first-order null X-points, and two variants of the exact configuration called snowflake-plus and snowflake-minus are often realized in steady-state, as shown in Fig. 1.

We implemented the world's first real-time SFD detection and control system on DIII-D in order to stabilize this configuration. The Plasma Control System (PCS) previously assumed that there is only a single isolated X-point in the search region. Iteration based complex search algorithms to find the multiple X-points is too time consuming to achieve in real-time and non-convergent solutions which may occur in these types of search algorithms 
is not acceptable for safe operation of DIII-D. Thus we employ a fast real-time deterministic snowflake identification algorithm, which accurately calculates two X-points (magnetic nulls) by locally expanding the magnetic equations. We assume that the plasma in the divertor region has a low beta, and the magnetic field there can be considered curl-free [1]. Then, the equation around the divertor is given as

$$
r \frac{\partial}{\partial r}\left(\frac{\partial \Psi}{\partial r}\right)+\frac{\partial^{2} \Psi}{\partial z^{2}}=0
$$

Here, $\Psi$ is the poloidal magnetic flux distribution in the $\mathrm{r}, \mathrm{z}$ plane that is found using the DIII-D real-time equilibrium reconstruction (rt-EFIT) in which the Grad-Shafranov (G-S) equations are solved [5]. This equation is rewritten in terms of dimensionless spatial variables $\delta r$ and $\delta z$ which are the radial and the vertical coordinates with respect to the snowflake center divided by the radial position of the snowflake center. Then $\Psi$ is expanded around the snowflake center in radial, $\delta r$, and vertical, $\delta z$, coordinates up to the third-order to achieve $\Psi_{\exp }=\Psi\left(c_{\text {exp }}, \delta r, \delta z\right)$, where $c_{\exp }$ are the expansion coefficients. A perfect snowflake divertor has a second-order null and it is necessary to go one order higher to analyze the formation; thus, a minimum of third-order expansion is necessary to quantify the SFD geometry. Solving the first two orders of the expanded equation (1) (i.e. finding conditions for the expansion coefficients such that the left hand side of both the $1^{\text {st }}$ and the $2^{\text {nd }}$ order equations equal to zero for all $\delta r$ and $\delta z$ ) and simplifying the expressions yields a reduced set of six unknown expansion coefficients. These unknowns are solved by first choosing three sample points (denoted with subscript sample) around the snowflake center and then evaluating the components of the magnetic field given as

$$
B_{\delta r_{\text {sample }}}=-\frac{1}{r_{\text {sample }}} \frac{\partial \Psi_{\text {exp }}\left(\delta r_{\text {sample }}, \delta z_{\text {sample }}\right)}{\partial \delta z_{\text {sample }}} \text { and } B_{\delta z_{\text {sample }}}=\frac{1}{r_{\text {sample }}} \frac{\partial \Psi_{\text {exp }}\left(\delta r_{\text {sample }}, \delta z_{\text {sample }}\right)}{\partial \delta x_{\text {sample }}} \text {, }
$$


where the left hand terms $B_{\delta r_{\text {sample }}}$ and $B_{\delta z_{\text {sample }}}$ are obtained from the rt-EFIT reconstruction. This gives us six linear equations for the six unknown expansion coefficients. These equations are solved using Gaussian elimination. Finally, the magnetic field nulls are solved by setting $B_{\delta r}=0, B_{\delta z}=0$. This results in the location of the two X-points: $\delta r_{X_{1}}\left(c_{\exp }\right), \delta z_{X_{1}}\left(c_{\exp }\right)$, $\delta r_{X_{2}}\left(c_{\text {exp }}\right)$, and $\delta z_{X_{2}}\left(c_{\text {exp }}\right)$. The resulting algorithm is a one-step (no-iteration) fast algorithm $(<<1 \mathrm{~ms})$ with reasonable accuracy $(<1 \mathrm{~cm})$, which has been implemented in the DIII-D PCS. This calculation is repeated in the PCS every 250 microseconds.

Once the locations of the two X-points are obtained, poloidal field (PF) coils are used to control the relative locations to obtain the desired SFD (exact, minus, plus). At DIII-D, the F4B, F5B, F8B are the PF coils closest to the divertor (see [Fig. 2(a)]) and they have therefore been used to control the SFD. F9B is also effective in the SFD manipulation. However, in order to avoid damage to the unprotected surfaces inside the cryopump, the strike point must be prevented from entering the cryopump gap under any possible circumstances. To satisfy this constraint without inducing any hardware or software protection, the F9B coil is set to be zero current.

The SFD control algorithm calculates the distance, $\rho$, and angle, $\theta$, [defined in Fig. 2(a)] components of the relative positions of the two X-points in addition to the radial and vertical coordinates of the snowflake centroid, $r_{c}$ and $z_{c}$, are shown with the blue cross in the Fig. 2(a). The values are compared to the user-requested values and the differences are filtered and fed to the control algorithm. For precise control, the effect of the change in PF coil currents, $\delta I_{P F}$, on the $\mathrm{X}$-point locations is calculated. This is achieved by applying the chain rule on the snowflake parameters: 


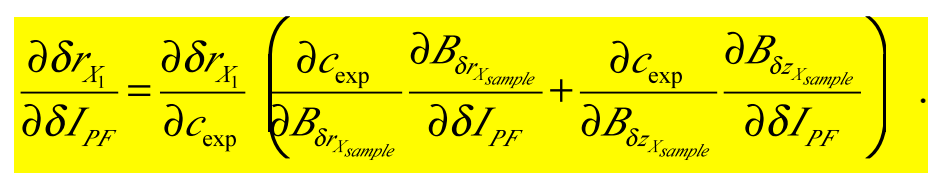

Here, the first two terms, $\frac{\partial \delta r_{X_{1}}}{\partial c_{\exp }}$ and $\frac{\partial c_{\exp }}{\partial B_{\delta r_{X_{s a m p l e}}}}$, are obtained through the manipulation of the G-S expansion, as explained above. $\frac{\partial B_{\delta r_{Y_{S a m p l e}}}}{\partial \delta I_{P F}}$ is found from the Green's Function of the G-S problem using the formulation stated in reference [5]. This enables us to write the variation of the snowflake geometric parameters in terms of the PF coil currents as

$$
\left[\begin{array}{l}
\delta \theta \\
\delta \rho \\
\delta r_{c} \\
\delta z_{c}
\end{array}\right]=A\left[\begin{array}{l}
\delta I_{F 4 B}- \\
\delta I_{F 5 B} \\
\delta I_{F 8 B-}
\end{array} .\right.
$$

Then, the control needed to achieve the requested snowflake configuration is obtained by taking the pseudo-inverse of this equation and multiplying it by a weighting function, $W$,

$$
\left[\begin{array}{l}
\delta I_{F 4 B} \\
\delta I_{F 5 B} \\
\delta I_{F 8 B}
\end{array}\right]=\left(A^{T} A\right)^{-1} A^{T} W\left[\begin{array}{l}
\delta \theta^{-} \\
\delta \rho \\
\delta r_{c} \\
\delta z_{c-}
\end{array} .\right.
$$

Since there are three actuators and four control parameters, the weighting function is used to define the importance of the parameters that we wish to control. Then, a ProportionalIntegral-Derivative control is experimentally tuned and used to obtain the requests to the power supplies to achieve the desired PF coil currents.

An example of an almost exact SFD obtained with the snowflake control is shown in Fig. 2, where the SFD control is turned on at 3 seconds (shown with the red line) and $\rho$ is 
controlled to a few $\mathrm{cm}$ until the end of the shot. Note that this is within the grid resolution of the rt-EFIT. As the perfect SFD is approached, broadening of the heat flux profile at the outer strike point is observed, as shown in Fig. 2(b).

This control enabled SFD minus, SFD plus, and exact SFD formations with varying $\sigma$, the distance between the X-points normalized to the minor radius, ranging from 0.08 to 0.5 in various scenarios. SFD was successfully integrated to an advanced tokamak (AT) scenario with normalized beta of $\beta_{\mathrm{N}}=3.0$ and $\mathrm{H}$-factor of $H_{98(y, 2)} \cong 1.35$, which is the energy confinement time normalized to the ITER98 $(y, 2)$ scaling. The flux profile for AT scenario with the standard divertor and SFD is shown in Fig. 3. We achieved a 2.5 times increase in the flux expansion and a 2.5 reduction in peak heat flux for many energy confinement times $(2-3$ s) without any adverse effects to the core plasma, such as confinement. The maximum allowable heat flux on plasma-facing tiles stipulate that SFD will operate under radiative conditions for fusion reactors. The radiative SFD regime was explored with gas seeding. Starting from attached conditions, plasma density was incrementally increased in consecutive plasma discharges in order to achieve detachment and radiative divertor conditions. SFD control was shown to be robust under partial and full detachment conditions during these scans.

\section{Detachment control}

Divertor "detachment", where the particle flux at the target plates drops by more than an order of magnitude, is achieved by increasing the density in the divertor. The ITER tokamak and future fusion reactors will require detached divertor plasmas to achieve acceptable divertor target heat loads [6]. However, it is difficult to stabilize this effect when plasmas become fully detached. The influx of impurities into the confined plasma cause high radiation levels from this region, which may result in the thermal instability of the whole plasma, 
known as Multi-faceted Asymmetric Radiation From the Edge (MARFE) [7]. Thus, ITER needs to operate in a state called partial-detachment under active feedback control in order to balance the need for acceptable divertor target heat loads and core stability.

We developed a new feedback control system on DIII-D to regulate and study the physics of divertor detachment. The system uses real-time electron temperature measurements from Thomson scattering, along with impurity line ratio measurement, to compute the location of the detachment front, while monitoring the core and divertor radiation measured by the bolometer diagnostic. We employed the new system to test the feasibility of envisioned ITER partial-detachment operation, using divertor Thomson measurements on DIII-D. (ITER will have a divertor Thomson with the diagnostic capability to measure as low as $1 \mathrm{eV}$ [8].) This control regulates the detachment front while minimizing the effect of the detachment on the core by fixing the core density independent of the detachment control. This is achieved by a feedback control system that uses two gas valves, as shown in Fig. 4. The valve close to the strike point regulates both deuterium fuel and impurity gas injection rates to maintain the detachment front (where the plasma temperature drops to less than a few eV) at a pre-set distance from the divertor target using the real-time electron temperature measurements, and the far-away valve keeps the core density stationary by using interferometry measurements.

The comparison of the core density, divertor density and divertor temperature of two DIII-D L-mode shots with and without detachment control is shown in Fig. 5. The feedback algorithm starts the control at 1.4 seconds. Detachment control increases the density at the divertor by a factor of $\sim 5$ and reduces the divertor temperature to $\sim 1-2 \mathrm{eV}$, while keeping the core density constant. The divertor and core conditions are kept roughly at a constant level throughout the controlled part of the shot (note that there is measurement noise from the divertor Thomson diagnostic). 
The control stabilized the detachment front fixed at the mid-distance between the strike point and the X-point throughout the shot, as shown in the 2D Thomson projection in Fig. 6. This partial detachment reduces the radiation peak from the strike point and spreads it across the detached area, as shown in Fig. 7. The new system allows the systematic study of the physics of plasma detachment and plasma-surface interactions under constant, reproducible conditions. The data from these experiments are used to test 2D models of the scrape-offlayer and divertor plasma as well as to interpret surface erosion and material migration measurements.

\section{Conclusions}

Two heat flux reduction control methods - SFD control and detachment control - were successfully implemented at DIII-D and integrated into advanced scenarios, thus aiding the development of heat management solutions that are needed for the next generation of fusion reactors. SFD control enabled the achievement of various SFD geometries and a $2.5 \mathrm{x}$ reduction in peak heat flux without any adverse effect to core plasma. Currently, the application of snowflake control at EAST tokamak is under development. This will allow us to address the erosion or material migration in a long-pulse machine, where major material challenges exist and the solutions can more easily be projected to future fusion reactors. Finally, a detachment control using the divertor Thomson measurements was shown to be able to keep the detachment front fixed at the mid distance between the strike point and the X-point throughout the shot. This approach may be a feasible control option for partialdetachment ITER scenarios.

\section{Acknowledgment}

This work was supported in part by the US Department of Energy under DE-AC0209CH11466, DE-AC52-07NA27344, DE-FC02-04ER54698, and DE-AC05-00OR22725. 
DIII-D data shown in this paper can be obtained in digital format by following the links at https://fusion.gat.com/global/D3D_DMP.

\section{References}

[1] D. Ryutov, Phys. Plasmas 14, 064502, 2007.

[2] Kotschenreuther M. et al, Phys. Plasmas 14, 072502, 2007.

[3] P. M. Valanju et al., Phys. Plasmas 16, 056110, 2009.

[4] B. LaBombard et al., "X-point target divertor concept and the Alcator DX high power divertor test facility", Bulletin of the American Physical Society, Volume 58, Number 16 (http://meetings.aps.org/link/BAPS.2013.DPP.CO4.2), 2013.

[5] J.R. Ferron et al., "Real time equilibrium reconstruction for tokamak discharge control", Nuclear Fusion 38, 1055-66, 1998.

[6] F. W. Perkins et al., "ITER Physics Basis”, Nuclear Fusion 39, 2137-2638, 1999.

[7] B. Lipschultz B et al., Nuclear Fusion 24, 977, 1984.

[8] E. Mukhin et al., J. of Instrumentation 7, C02063, 2012.

\section{List of Figure Captions}

Fig. 1. Three plasma equilibria in DIII-D showing the exact snowflake configuration with a double null; the snowflake-minus configuration with the secondary X-point in the private flux region; and the snowflake-plus configuration with the secondary X-point in the SOL.

Fig. 2. (a) PF coils used in SFD control and the definition of the SFD configuration parameters. (b) Plasma controlled to almost exact SFD. The SFD control starts at $3000 \mathrm{~ms}$. The lower panel shows the heat flux at the outer strike point (\#155478). 
Fig. 3. The heat flux profile at the inner and outer strike point for (a) the standard divertor double null AT and (b) the SFD (-) double null AT. Detail of the SFD geometry is shown in inset (c).

Fig. 4. Sketch of the partial-detachment control system.

Fig. 5. Data showing feedback control of divertor detachment. Red-detachment feedback control on (\#153816). Black - detachment control off (no divertor fueling - \#153814). Top: Line average core density Middle: Divertor density measured by divertor Thomson $(\sim 2$ $\mathrm{cm}$ above the floor $)$. Bottom: Divertor temperature measured by divertor Thomson $(\sim 2 \mathrm{~cm}$ above the floor).

Fig. 6. 2D Projected divertor Thomson temperature measurements for DIII-D: (a) shot without detachment control (\#153814) shows no detachment, (b) shot with partialdetachment control (\#153816) achieves detached cold front region, shown in purple and blue. Note that for visualization purposes the color map is cut-off at $20 \mathrm{eV}$ and all higher temperature measurements are shown in red.

Figure 7. Radiation profile around the divertor for DIII-D: (a) shot without detachment control (\#153814) shows peaked radiation from the strike point, (b) shot with partialdetachment control (\#153816) spreads the radiation across the detached area. 

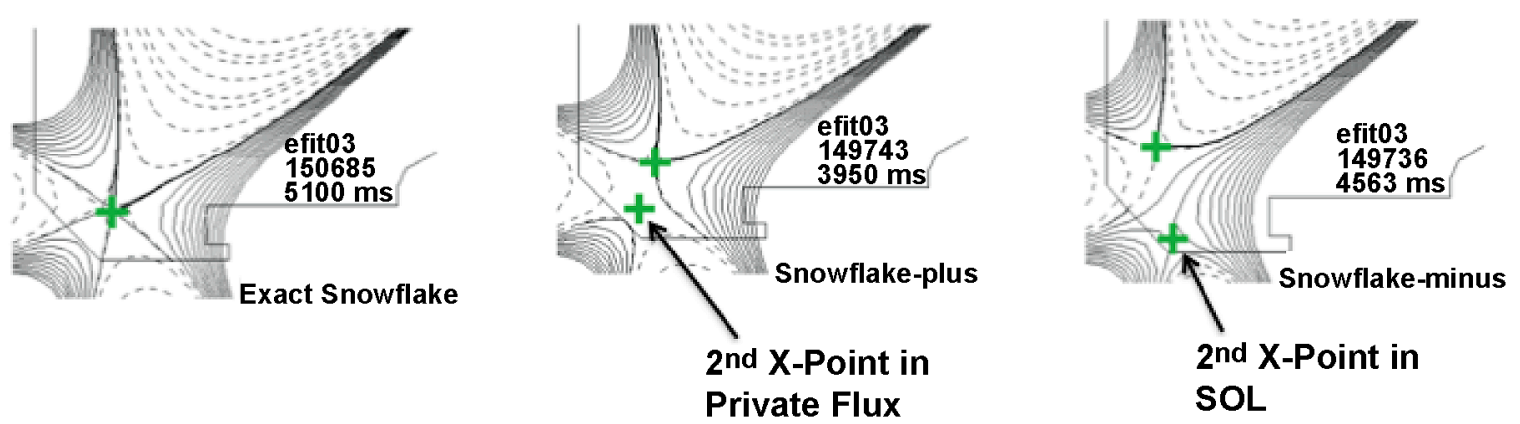

Fig. 1 


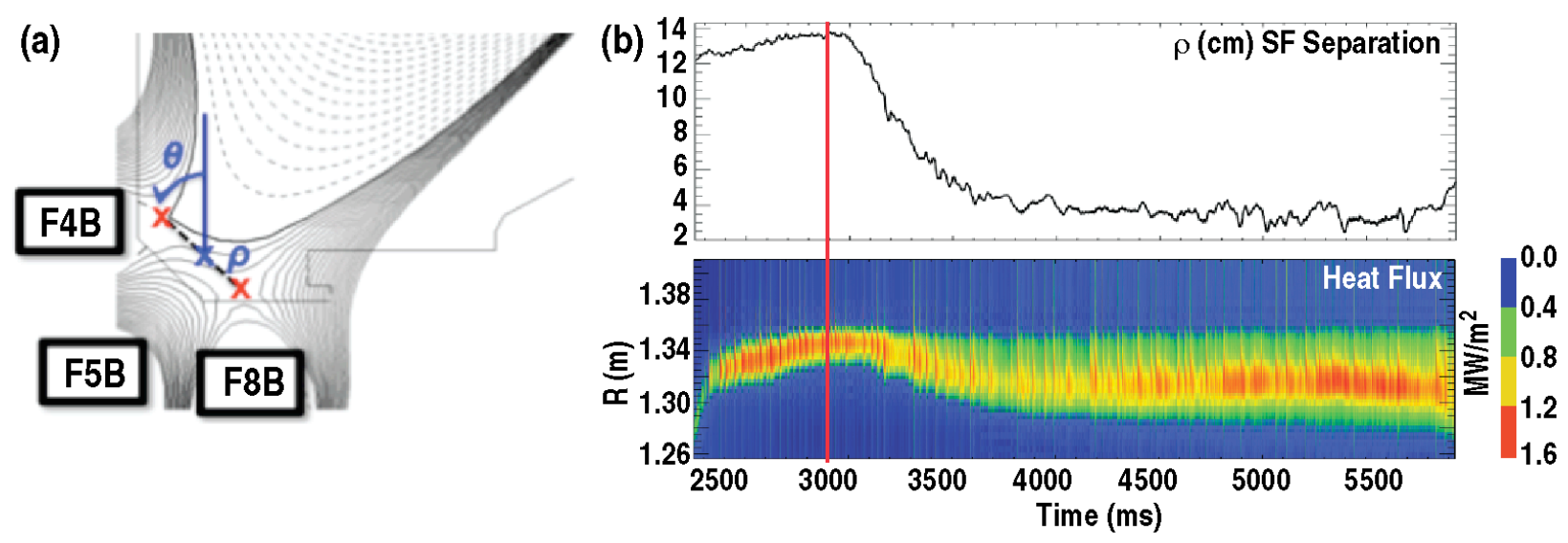

Fig. 2 

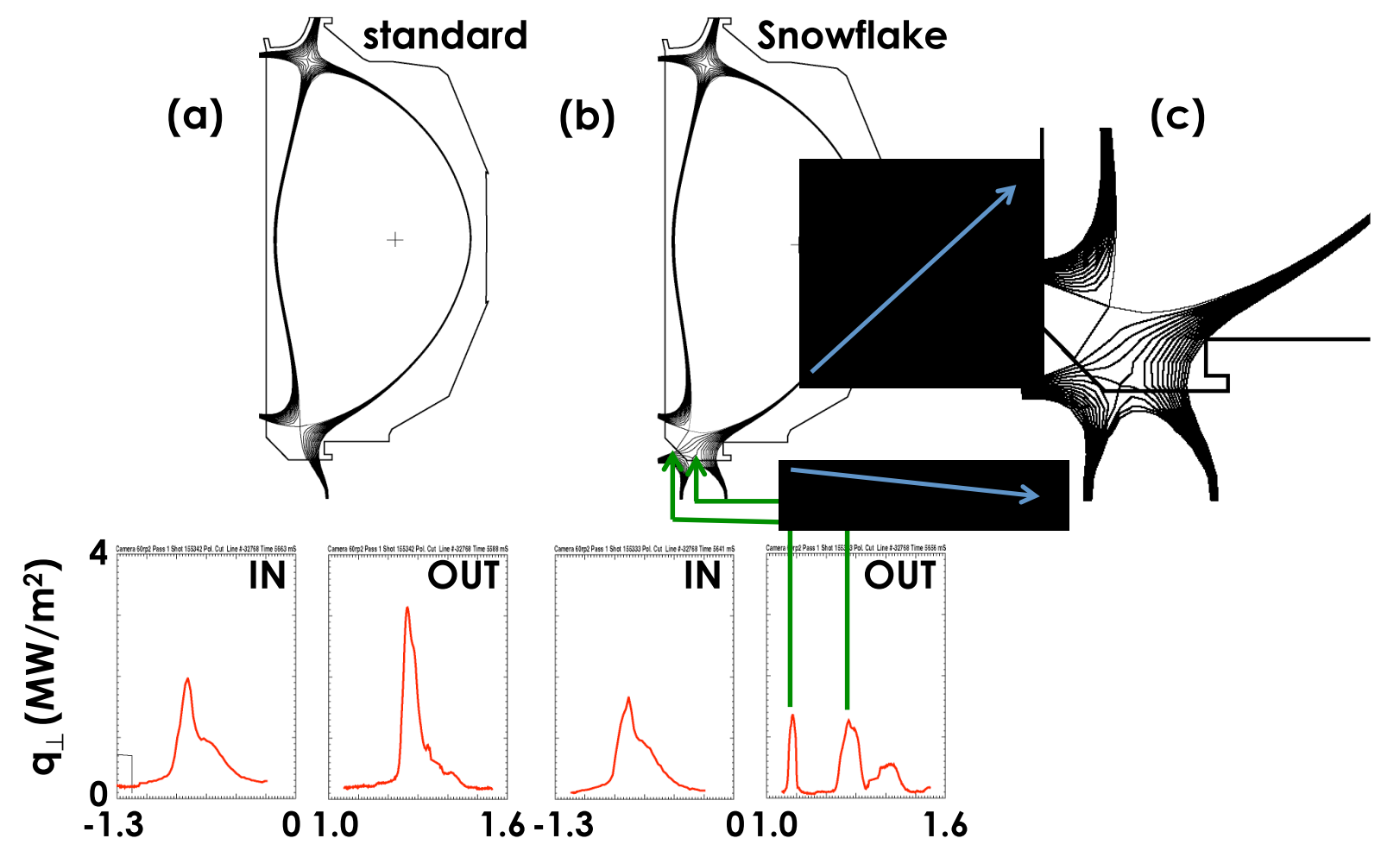

Fig. 3 


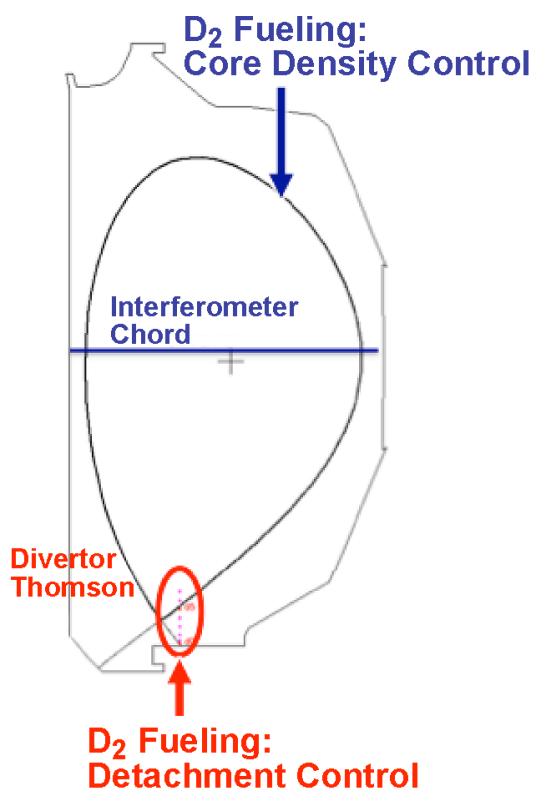

Fig. 4 


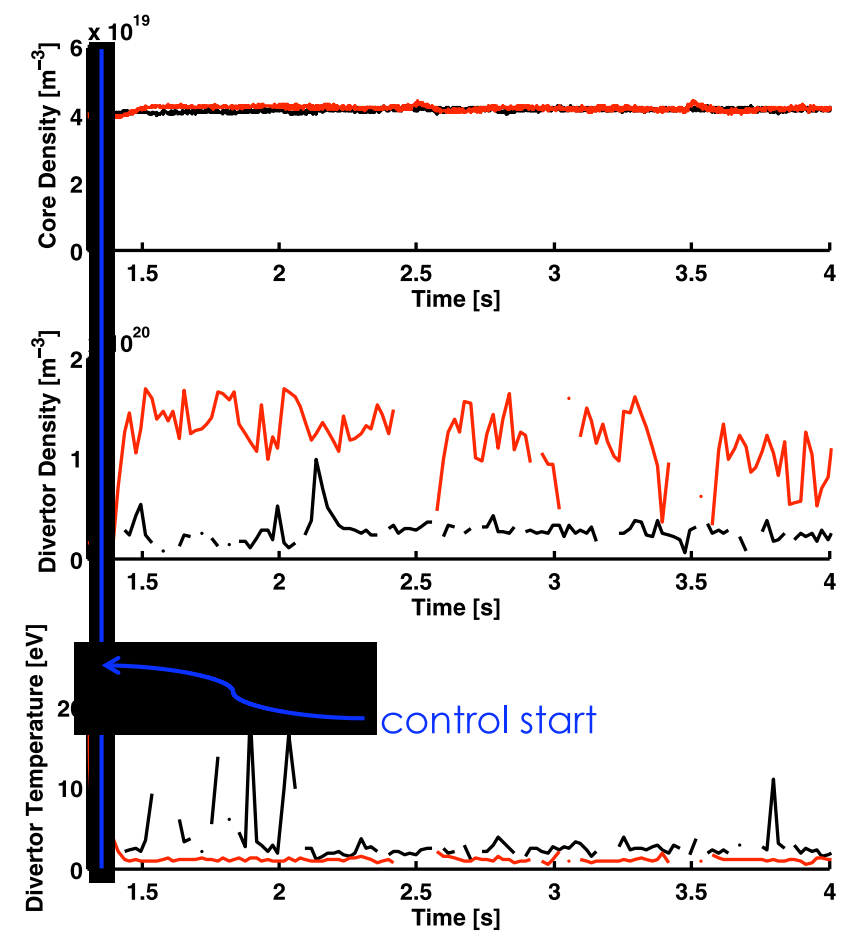

Fig. 5 


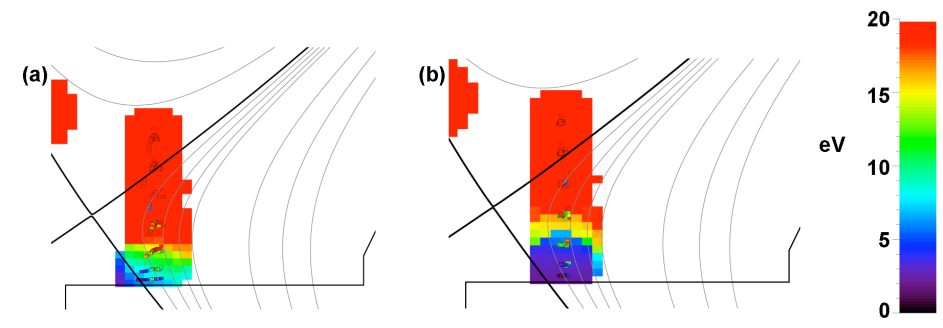

Fig. 6 


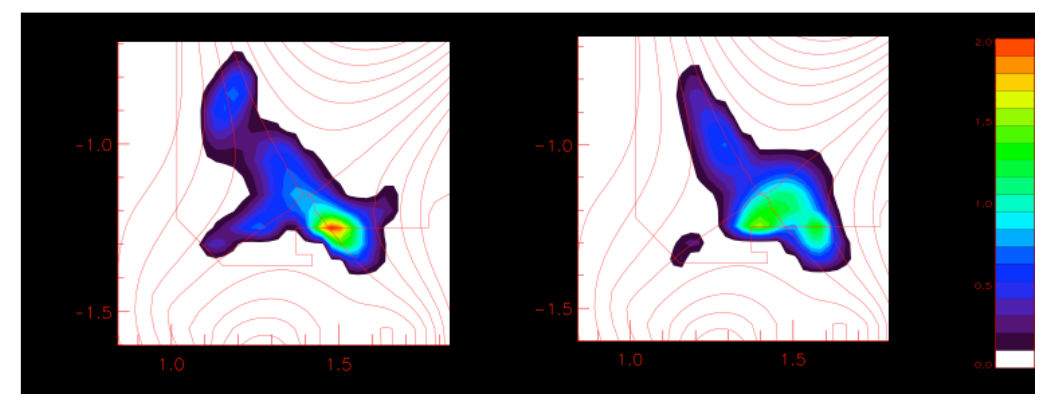

Fig. 7. 